\title{
OBSERVED MASS LOSS FROM CENTRAL STARS OF
}

\section{PLANETARY NEBULAE}

\author{
M. PERINOTTO \\ Dipartimento di Astronomia e Scienza dello Spazio, Universita' de Firenze
}

\begin{abstract}
In the Introduction we recall the mass loss history of a progenitor of a planetary nebula (PN). Then we concentrate on the status of knowledge of fast winds in central stars of planetary nebulae (CSPN) : the detection and statistics, the observed edge velocities, relationships of the edge velocities with other stellar or nebular parameters. We then summarize the methods used to derive the mass loss rates associated to the fast winds, and review the determinations of the "observed" mass loss rates. The comparison with predictions from the radiation driven theory (RDT) is then discussed as well as possible lines for future improvements.
\end{abstract}

\section{Introduction}

The general trend of the mass loss phenomenon along the life-time of a single star progenitor of a PN was clear already at the last IAU Symposium on PNe, five years ago, in Mexico city. Progress has been made meanwhile which helps to put in a better perspective some aspects of the subject, but much work needs still to be made before we reach a detailed quantitative understanding of the whole topic.

The wind phenomenon can be divided in various phases following the evolution of the star across the HR diagram. We can distinguish four main phases, each possibly with its own dominant mechanism for the production of the wind. Within each phase, some sub-phases might be identified from the specific stellar structure, which implies particular properties for the outher layers too, and consequently for the character of the wind. We have, in sequence: phase I, with sub-phases Ia (first Red Giant Branch), Ib (Horizontal Branch), Ic (early Asymptotic Giant Branch); phase II, with subphases IIa (pulsed Asymptotic Giant Branch), and IIb (transition from the AGB up to the end of the "superwind" phase; phase III, which represents the remaining transition up to the appearance of the optical nebula and phase IV, with sub-phases IVa (stellar luminosity approximately constant from the value at the end of the ABG phase up to 0.9 of it) and IVb (fading phase : stellar luminosity up to 0.1 of the final value on the AGB).

In phase I the wind velocity is around $10 \mathrm{~km} / \mathrm{s}$ and the mass loss rate is believed to be represented by the Reimers $(1975 \mathrm{a}, \mathrm{b})$ law : $\mathrm{dM} / \mathrm{dt}=\eta 4.010^{-13} \mathrm{LR} / \mathrm{M}$, where $\mathrm{L}, \mathrm{R}$ and $\mathrm{M}$ are the usual stellar quantities in solar units, and $\dot{M}$ is in solar masses per year. The law has been obtained from some $\mathrm{K}$ and $\mathrm{M}$ giants and supergiants, with $\eta$ ranging from 0.3 to 3 . Subsequent studies proved that that in Population II red giants $\eta$ can be set equal to $0.4 \pm 0.04$, while equally stringent limits are not easy to set for intermediate-mass stars (cf. Iben and Renzini, 1983) and then in general for the progenitors of $\mathrm{PNe}$.

In phase II the wind becomes quite stronger, the so called "superwind", whose canonical $\dot{M}$ is of $10^{-4} \mathrm{Mo} \mathrm{yr}^{-1}$ for a duration of 1000 years. These values are 
requested to match the nebular mass observed in the $\mathrm{PN}$ of well known distance K 648 in M 15 (Renzini, 1981). The well known OH/IR sources are believed to represent this phase (see Habing, these Proceedings).

To the subsequent phase III (transition typically from a stellar temperatures of $\mathrm{T}=5000 \mathrm{~K}$ to $\mathrm{T}=30,000 \mathrm{~K}$ ), the Proto-PN objects (see Kwok, these Proceedings) and the optically bright Post-AGB stars (cf. Trams, 1992) belong.

Finally in phase IV, with the regular optically visible nebula coming out, the central star exhibits a wind much faster than in the previous phases : it is called the "fast" wind.

The importance of the winds in these various phases for the evolution of the central stars of planetary nebulae and for the relationship with the presently observed nebulae and with their history has been widely recognized. A great deal of work has been recently made and is currently pursed in the specific area of the hydrodynamics of the interaction of the various type of winds, with the aim of explaining the morphology in "groups" of PNe as well as in individual objects (see the review by Balick in these Proceedings). To this subject a precise knowledge of the fast wind parameters, in particular of the mass loss rate, is crucial.

\section{The fast winds}

The information on these winds in the CSPN come essentially from P Cygni profiles of resonance or excited lines of heavy ions observed in the UV range from the IUE satellite, with the addition of some $\mathrm{P}$ Cygni-like profiles observed in the optical range mainly in stars of $\mathrm{W}$ - $\mathrm{R}$ type.

\subsection{DETECTION AND STATISTICS}

A recent statistical study of the occurence of winds in CSPN has been made by Patriarchi and Perinotto (1991). Over a total of 159 objects examined, 81 showed a stellar continuum, thus giving the possibility to detect $\mathrm{P}$ Cygni profiles. Of these, 45 CSPN exhibited a fast wind, while 29 did not and 7 cases resulted to be doubtful. Thus about $60 \%$ of the examined CSPN did show a wind a $40 \%$ did not, to the level of detectability of the IUE spectra.

The occurence of the wind has been found to depend on the stellar gravity (Cerruti-Sola and Perinotto, 1985), in the sense that CSPN with gravity smaller than $\log g=5.2$ (cgs units) almost always have a wind, while that is not so for the higher gravity CSPN, and on the luminosity (Pauldrach et al., 1988), so that the more a CSPN departs from the Eddigton luminosity, less frequent is the occurence of the wind.

\subsection{EDGE VELOCITIES}

A list of edge velocities in CSPN has been published by Patriarchi and Perinotto (1991), in connection with a study of the expansion velocities of the nebulae. To complete and update that list, the following values of Vedge are to be considered : $2700,1700,1550,1350 \mathrm{Km} / \mathrm{s}$ in NGC $6210,6826,6891$ and IC 4593 , respectively, 
by Bianchi and De Francesco (these Proceedings), $3600 \mathrm{~km} / \mathrm{s}$ in NGC 6905 (Heap, 1986), 1000-2000 km/s in He 2-99 (Kaler et al., 1989), $1800 \mathrm{~km} / \mathrm{s}$ in Hu 2-1 (CerrutiSola and Perinotto, 1985), $2150 \mathrm{~km} / \mathrm{s}$ in PK $75+351$ (Feibelman and Bruhweiler, 1989).

These values come from high resolution IUE spectra, a part from those of $\mathrm{He}$ 2-99 and $\mathrm{Hu} 2-1$, where low resolution IUE specira had to be used. Moreover there is a value of about $500 \mathrm{~km} / \mathrm{s}$ in NGC 6302 by Meaburn and Walsh (1980) from optical data. In NGC 6826 there is another determination by Altner et al. (these Proceedings), equal to $1250 \mathrm{~km} / \mathrm{s}$, much lower than the above mentioned value, which is close to the one listed by Patriarchi and Perinotto, equal to $1600 \mathrm{~km} / \mathrm{s}$. This new determination differs from the previous ones quite more than the usual error from high resolution IUE spectra (about $100 \mathrm{~km} / \mathrm{s}$ ) and was obtained from the same high resolution IUE spectra used e.g. by Perinotto et al. (1989), who found Vedge $=1750 \mathrm{~km} / \mathrm{s}$ and Vterminal $=1665 \mathrm{~km} / \mathrm{s}$. Looking back to the original profiles, in particular to the well defined profile of the CIV 1550 A line, we judge that a Vedge of $1560 \mathrm{~km} / \mathrm{s}$ is in fact more realistic. This however is still unconfortably far from the $1250 \mathrm{~km} / \mathrm{s}$ by Altner et al.. This object deserves then further attention.

The observed values of Vedge range from about 600 to $3600 \mathrm{~km} / \mathrm{s}$.

\subsection{RELATIONSHIP OF Vedge WITH STELLAR AND NEBULAR PARAMETERS}

An important, almost linear, relationship between the edge velocity of the stellar winds and the temperature of the central stars was found by Heap (1986). We have added 9 new objects to the 18 shown in her Fig.3. The new objects follow well the trend of the previous ones, so that the best fit obtained by Heap is confirmed by the new observations. Her interpretation of the relationship is thus strengthened : stars of similar mass ( 0.6 solar masses) evolve in the $\mathrm{HR}$ diagram at constant luminosity. The radius then decreases, the gravity increases and then also the escape velocity increases. That implies, according to the prediction of the radiation driven theory, an higher velocity of the wind. As a consequence, this relationship supports the radiation driven theory explanation for the fast winds in CSPN.

A similar relationship exists also for the O-B stars of Population I, where the RDT is firmly considered to provide the dominant mechanism for the production of the winds.

Another, almost linear, relationship has been found by Patriarchi and Perinotto (1991). That is between the edge velocity of the stellar winds and the expansion velocity of the associated optical nebula. Although the scatter is large, the authors argued that the relationship is consistent with the multiple-wind interacting theory, in the adiabatic case. Basically a larger Vedge implies an higher temperature of the shocked region and therefore a larger pressure on the previously ejected matter (see also the review by Heap in these Proceedings).

\section{The mass loss rate}

We quicly recall the methods used to derive the mass loss rates associated with the fast winds in CSPN, review the determinations made after the Mexico city 
Symposium, focussing on the "observed" values, compare the observed values with those predicted by the radiation driven theory and present some suggestions for future work.

\subsection{METHODS TO DERIVE THE MASS LOSS RATE}

One can distinguish between "theoretical" and "semi-empirical" methods. In both cases the calculated P Cygni profiles must match the observed ones.

In the first type the mechanism(s) responsible for the production of the wind is (are) specified, while that is not so in the second type of methods. The mass loss rates coming from the last methods are called "observed" $\dot{M}$.

The theoretical methods are clearly more satisfactory and would give the best answer, provided one is able to include all the necessary physics. The dominant mechanism is recognized to be the pressure by radiation on the heavy ions. The status of art in this area is reviewed by Kudritzki (these Proceedings).

In the semi-empirical methods the velocity law is assumed and used as a parameter to fit the observations. The various semi-empirical methods then differ in the physical description of the phenomenon.

Basically the following approximations and approaches enter: 1a) Sobolev approximation for the opacity, or $1 \mathrm{~b}$ ) No Sobolev approximation; $2 \mathrm{a}$ ) transfer equation in the observer's frame or $2 \mathrm{~b}$ ) in the comoving frame with the fluid; $3 \mathrm{a}$ ) two-levels atom or $3 \mathrm{~b}$ ) multi-level atom; $4 \mathrm{a}$ ) interaction among the components of multiplets not handled or rougly handled or 4b) correctly handled; $5 \mathrm{a}$ ) behaviour of the opacity across the fluid assumed (and treated as a parameter) or $5 \mathrm{~b}$ ) occupation number$\mathrm{s}$ derived from statistical equilibrium calculations; $6 \mathrm{a}$ ) trend of the temperature across the wind assumed or $6 \mathrm{~b}$ ) derived from the solution of the energy balance; $7 \mathrm{a}$ ) turbulence in the wind not considered or $7 \mathrm{~b}$ ) handled in some way; $8 \mathrm{a}$ ) role of the underlying photospheric lines in the transfer considered or $8 \mathrm{~b}$ ) not taken into account; $9 \mathrm{a}$ ) source function purely radiative or $9 \mathrm{~b}$ ) able to account for collisional effects; 10b) use of the full shape of the observed profile in the comparison with the observations or 10a) of some quantity derived from the profile, as the first moment of the flux distribution (which can be obtained also from spectra of resolution close to the instrumental resolution).

It is evident that a "b" condition specifies a superior method. The methods actually used to derive "observed" $\dot{M}$ in CSPN have been summarized by Perinotto (1989) : i) the escape probability method (EP), and the same with improvements by Olson $(\mathrm{EPm})$, ii) the first moment of the flux distribution method (FM), and the same with modifications by Surdej (FMm), iii) the comoving frame method (CF) and iv) the SEI method (Sobolev plus exact integration). To these we must now add : v) the comoving frame method with statistical equilibrium equations across the wind solved in multi-level atom (CFS), used by Werner and Koesterke (1992).

Relative to the $(\mathrm{a}, \mathrm{b})$ conditions above illustrated, the various methods use "a" conditions except for the following " $b$ " conditions. Method EP: 10; method FM : none and in addition the opacity law is not treated as a parameter but is fixed to some 'average' behaviour; method CF : 1,2,4,10; method SEI : 4,7,8,9,10 and as far as 1 is considered, something of quality between a and b. In the CFS method 
essentially all conditions are of type "b".

To the discussion of the merit of the various methods by Perinotto (1989), we add here the following. The EP method has been overcome by the SEI method (which has been widely distributed and is available upon request). The FM (or FMm) method may still be useful when spectra of resolution comparable with the instrumental resolution are available, but is of limited values because both the velocity and the opacity laws are assumed equal to some average shapes. The CF method is good, particularly for the regions of the wind closer to the star, but it has various limitations as is clear from its "a" conditions. At present a rather good method is the SEI method, while the most powerful one is the CFS method.

\subsection{OBSERVED MASS LOSS RATES}

In a review on the same subject at the Symposium of Mexico city, (Perinotto, 1989), I reported the determinations of the observed mass loss rates obtained by that time.

They referred to $20 \mathrm{CSPN}$ studied with low or high resolution IUE spectra using the methods mentioned in the previous Section. They ranged between $2.10^{-6}$ and $5 \cdot 10^{-11}{\mathrm{Mo} \mathrm{yr}^{-1}}$, but values by different authors in the same objects did scatter by 1-2 order of magnitude, due to the different methods and to the different values of the fundamental stellar parameters adopted.

Since then the following studies have been made: A reanalysis by Hutsemekers and Surdej (1989) with their FMm method of 14 CSPN previously studied with low resolution IUE spectra and the FM method by Cerruti-Sola and Perinotto (1985); a study of five CSPN with the SEI method by Cerruti-Sola and Perinotto (1989) plus studies of the following individual objects : A78 by Kaler et al. (1988) with the FM method and by Werner and Koesterke (1992) with the CFS method; PK 75 +35 1 by Feibelman and Bruhweiler (1989) with the FM method and NGC 40 by Bianchi (1992) with the EPm method plus another approach based on the optical helium lines of WR stars.

These determinations are not of the same accuracy for what explained before.

To these one should add the preliminary values of $\dot{M}$ presented at this meeting of IC 2149 and Tc 1 by Modigliani et al. (these Proceedings) and of NGC 6210, 6826, 6891, IC 4593 by Bianchi and De Francesco (these Proceedings), in both cases with the SEI method.

We report in Table 1 the objects that we feel have at present the best observed $\dot{M}$ : they have been studied by Perinotto et al. (1989) and by Cerruti-Sola and Perinotto (1989) with the SEI method and by Werner and Koesterke with the CFS method. 


\begin{tabular}{||r|r|l||r|r||r|r|r||}
\hline Object & $\mathrm{T} / 1000(\mathrm{~K})$ & $\mathrm{R} / \mathrm{R} \odot$ & \multicolumn{3}{|c||}{ Velocity $(\mathrm{km} / \mathrm{s})$} & \multicolumn{3}{|c||}{$d M / d t\left(M \circ y r^{-1}\right)$} \\
\hline & & & V(term. $)$ & V(turb. $)$ & Obs. & RDT & Reim. \\
\hline \hline NGC 1535 & 77 & 0.55 & 1900 & 95 & $1.4 \mathrm{e}-9$ & $9.9 \mathrm{e}-9$ & $3.4 \mathrm{e}-9$ \\
6210 & 90 & 0.29 & 2180 & 110 & $2.2 \mathrm{e}-9$ & $2.9 \mathrm{e}-9$ & $1.0 \mathrm{e}-9$ \\
6543 & 60 & 0.70 & 1900 & 15 & $4.0 \mathrm{e}-8$ & $4.0 \mathrm{e}-9$ & $2.6 \mathrm{e}-9$ \\
6826 & 45 & 1.45 & 1750 & 90 & $6.4 \mathrm{e}-8$ & $7.5 \mathrm{e}-9$ & $7.3 \mathrm{e}-9$ \\
7009 & 88 & 0.45 & 2770 & 30 & $2.8 \mathrm{e}-9$ & $1.3 \mathrm{e}-8$ & $3.2 \mathrm{e}-9$ \\
IC 418 & 37 & 2.8 & 940 & 45 & $6.3 \mathrm{e}-9$ & $2.2 \mathrm{e}-8$ & $2.3 \mathrm{e}-8$ \\
IC 4593 & 35 & 2.2 & 1000 & 100 & $4.2 \mathrm{e}-8$ & $5.0 \mathrm{e}-9$ & $8.7 \mathrm{e}-9$ \\
A 78 & 115 & 0.24 & 3700 & - & $2.5 \mathrm{e}-8$ & $7.7 \mathrm{e}-9$ & $1.3 \mathrm{e}-9$ \\
\hline \hline
\end{tabular}

The accuracy of the observed $\dot{M}$ is estimated to be within factors $3-5$. That of the values from the radiation driven theory, as calculated here (see the next Section), is not easy to judge, but should be higher, particularly if the correct mass of the individual objects is used.

\subsection{COMPARISON WITH PREDICTION BY THE RADIATION DRIVEN THEORY}

In Table 1 we also report the values predicted by the radiation driven theory, calculated with the analytical formulation by Kudritzki et al. (1989) using the latest prescriptions of the force multiplier parameters for CSPN by Kudritzki et al. (1992), assuming the same mass of 0.6 Mo for all the central stars.

The calculated and the observed values are to within a factor of 10 , apparently with no systematic effects. In the last column of Table 1 we give the values of $M$ resulting from the Reimers formula (with $\eta=1$ ) (see Introduction). These numbers are close to within a small factor to those from the radiation driven theory. That is surprising, considering the origin of the Reimers parametrization.

\section{Conclusions and suggestions for future work}

From the comparison made in the previous Section and considering the errors of the methods used to obtain the mass loss rates of fast winds in CSPN, one might conclude that the semi-empirical methods have been useful to prove the substantial correctness of the theoretical methods, based on the pressure of radiation on the heavy ions. And one might also conclude to be advisable for the future to rely only upon theoretical determinations of the mass loss rates.

We believe instead that there are reasons that justify the opportunity to continue also the work with the semi-empirical methods. First the correctness of the theoretical methods has to be proved on the full range of the fundamental stellar parameters, second it is well known that radiative winds are highly unstable (cf. Castor, 1991).

That means on one side that slightly different physical conditions in the subsonictransonic regions of the wind, may produce rather different mass loss rates in stars which, generally speaking, are very similar. And, on another side, that at different epocs the mass loss rate of the same star might be different.

Moreover the present level of the radiation driven theory in hot stars needs still improvements (cf. Lamers, 1991), which are under way (see Kudritzki, these 
Proceedings).

Then we believe that semi-empirical methods remain important for the understanding of the winds in CSPN and for our knowledge of their mass loss rates.

Improvements for a better measurement of this quantity include : a) a search for the variability in the winds; b) a better determination of the stellar radius (then of the distance, e.g. from the coming Hipparcos data), of the stellar temperature (via better atmospheric models and space experiments in the EUV spectra range) and of the chemical composition in the wind; c) a more precise definition of the observed p Cygni profiles and of the stellar continuum, properly accountign for the numerous spectral features; d) the use of other wind signatures (in addition to the UV P Cygni profiles) as optical P Cygni profiles and hopefully of the free-free wind continuum in the radio and infrared spectral ranges.

Clearly we need to observe at a sufficient spectral resolution more objects than those observable with IUE, and therefore we need to use the Hubble Space Telescope.

\section{REFERENCES}

Bianchi,L.: 1992, Astron. Astrophys. 253, 447.

Castor,J.I.: 1991, in Stellar Atmospheres: Beyond Classical Models, eds. L.Crivellari,I.Hubeny,D.G.Hummer, Kluwer Academic Publ., Dordrecht,p. 221.

Cerruti-Sola,M.,Perinotto,M.: 1985, Astrophys. J. 291, 237.

Cerruti-Sola,M.,Perinotto,M.: 1989, Astrophys. J. 345, 339.

Feibelman,W.A.,Bruhweiler,F.C.: 1989, Astrophys. J. 347, 901.

Heap,S.: 1986, in New Insights in Astrophysics, ESA SP-263, p. 291.

Hutsemekers,D.,Surdej,J.: 1989, Astron. Astrophys. 219, 237.

Iben,I.Jr.,Renzini,A.: 1983, Ann. Rev. Astron. Astrophys. 21, 271.

Kaler,J.B.,Feibelman,W.A.,Henrichs,H.F.: 1988, Astrophys. J. 324, 528.

Kaler,J.B.,Shaw,R.A.,Feibelman,W.A.,Lutz,J.H.: 1989, Astrophys. J. Suppl. 70, 213.

Kudritzki,R.P.,Pauldrach,A.W.A.,Puls,J.,Abbott,D.C.: 1989, Astron. Astrophys. 219, 205.

Kudritzki,R.P.,Hummer,D.G.,Pauldrach,A.W.A.,Puls,J., Najarro, F.,Imhoff,J.: 1992, Astron. Astrophys. 257, 655.

Lamers,H.J.G.L.M.: 1991, in Stellar Atmospheres: Beyond Classical Models, NATO ASI Ser. Conference, eds. L.Crivellari, I.Hubeny, D.G.Hummer, Kluwer Academic Publ., Dordrecht,p. 311.

Meaburn,J.,Walsh,J.R.: 1980, Mon. Not. R. astr. Soc. 191, 5p.

Patriarchi,P.,Perinotto,M.: 1991, Astron. Astrophys. Suppl. 91, 325.

Pauldrach,A.W.A.,Puls,J.,Kudritzki.R.P.,Mendez,R.H.,Heap,S.R.: 1988, Astron. Astrophys. 207, 123.

Perinotto,M.: in IAU Symp. N. 131, Planetary Nebulae, ed. S.Torres-Peimbert, Reidel Publ., Dordrecht,p. 293.

Perinotto,M.,Cerruti-Sola,M.,Lamers,H.J.G.L.M.: 1989, Astrophys. J. 337, 382.

Reimers,D.: 1975a, Mem. Soc. R. Sci. Liege, 6 Ser., 8, 369. 
Reimers,D.: 1975b, in Problems in Stellar Atmospheres and Envelopes, eds. B.Baschek,W.H.Kegel,G.Traving, Springer Publ., p. 229.

Renzini,A.: 1981, in Physical Processes in Red Giants, eds. I.Iben Jr., A.Renzini, Reidel Publ., Dordrecht,p. 165.

Trams,N.R.: 1992, Thesis, Utrecht, SRON Laboratorium.

Werner,K.,Koesterke,L.: 1992, in The Atmospheres of the Early-Type Stars, eds. U.Heber,C.S.Jeffery, Springer-Verlag, Berlin,p. 288. 